\title{
Increasing PACE of PACEMAKER Imaging!
}

\author{
June A Yamrozik ${ }^{1 *}$, Mark Doyle ${ }^{1}$, Ronald B Williams ${ }^{1}$, Geetha Rayarao ${ }^{1}$, Diane V Thompson ${ }^{1}$, Moneal Shah', \\ Huma Samar ${ }^{2}$, Robert W Biederman ${ }^{1}$ \\ From 19th Annual SCMR Scientific Sessions \\ Los Angeles, CA, USA. 27-30 January 2016
}

\section{Background}

Pacemaker/ AICD imaging is currently being done on patients in the MRI environment. A vigilant team consisting of the (Cardiologist, EP staff, and technologists) with close patient monitoring and supervision is making such an unmentionable procedure a success. However, is the diagnosis from imaging these patients adding valuable irrefutable information to merit such a risk?

\section{Methods}

A total of 157 patients were imaged on a GE CV/i Excite Version 12, $1.5 \mathrm{~T}$ system (GE, Milwaukee, WI). 31 patients had an AICD, 13 patients had an AICD/Pacemaker, 6 patients had a single pacemaker lead, 14 REVO pacemakers and 93 patients had a complete pacemaker implantation. A specific criteria was followed for all the patients undergoing this procedure to scrutinize if the final diagnosis provided additional information in patient care. A checklist of three questions were gathered and answered after the final interpretation of the MRI.

1 . Does the diagnosis change?

2. Does the MRI provide additional information to the existing diagnosis?

3. Does patient management change?

If yes was answered any of the above questions it was considered that the MRI scan was of value to patient diagnosis.

\section{Results}

All patients completed the procedure with no adverse events and the pacemaker was interrogated after the procedure by EP Lab and reprogrammed under the direction of the Cardiologist. The average MRI scan time was $20 \pm 55 \mathrm{~min}$. A total of 157 patients imaged, 114 (73\%) were neurology cases, 7 musculoskeletal (4\%) and 36(23\%) were cardiac/vascular cases.

${ }^{1}$ Cardiac MRI, Allegheny General Hospital, Pittsburgh, PA, USA

Full list of author information is available at the end of the article
After reviewing the results from the 114 neurology cases $21(18 \%)$ out of the 114 showed that the MRI not only provided additional information but changed the original diagnosis and in turn their course of medical treatment. 79 patients (69\%) provided additional information to the diagnosis. Thus a total of 100 patients (88\%) showed that the MRI scan was of value to the final diagnosis. 14(12\%) out of the 114 patients imaged did not provide any further information but confirmed the original diagnosis. The 36 cardiac cases showed in 5 patients (14\%) the MRI provided additional information to change the original diagnosis and also patient management. 28 (77\%) showed that extra information was gathered. 3(8\%) were unable to interpret due to artifact from AICD device. In essence, $92 \%$ of the Cardiac population benefited by having the MRI done. The 7 musculoskeletal demonstrated that $6(85 \%)$ provided additional information and $1(15 \%)$ changed patient management

\section{Conclusions}

The use of PM/AICD imaging in MRI remains controversial but with a cautious cardiac team increased confidence in its use is found. Herein, we show that MRI procedures on carefully selected patients with pacemakers/AICD's are beneficial and substantially add valuable irrefutable information to patient diagnosis and management. We propose that not only are Pacemakers/AICD's no longer taboo in the MRI environment but they can be markedly efficient with life-altering and life-saving consequences.

\section{Authors' details \\ ${ }^{1}$ Cardiac MRI, Allegheny General Hospital, Pittsburgh, PA, USA. ${ }^{2}$ Cardiology, VA Medical Center, Loma,Linda, CA, USA.}

Published: 27 January 2016

doi:10.1186/1532-429X-18-S1-T6

Cite this article as: Yamrozik et al:: Increasing PACE of PACEMAKER

Imaging!. Journal of Cardiovascular Magnetic Resonance 2016 18(Suppl 1):T6.
C Biomed Central

(c) 2016 Yamrozik et al. This is an Open Access article distributed under the terms of the Creative Commons Attribution License (http:// creativecommons.org/licenses/by/4.0), which permits unrestricted use, distribution, and reproduction in any medium, provided the original work is properly cited. The Creative Commons Public Domain Dedication waiver (http://creativecommons.org/publicdomain/ zero/1.0/) applies to the data made available in this article, unless otherwise stated. 